\title{
Separable and semiparametric network-based counting processes applied to the international combat aircraft trades
}

\author{
Cornelius Fritz ${ }^{1, *}$ (D), Paul W. Thurner ${ }^{2}$ and Göran Kauermann ${ }^{1}$ \\ ${ }^{1}$ Department of Statistics, LMU Munich, Munich, Germany (e-mail: goeran.kauermann@stat.uni-muenchen.de) and \\ ${ }^{2}$ Geschwister Scholl Institute of Political Science, LMU Munich, Munich, Germany (e-mail: \\ paul.thurner@gsi.uni-muenchen.de) \\ *Corresponding author. Email: cornelius.fritz@stat.uni-muenchen.de
}

Action Editor: Stanley Wasserman

\begin{abstract}
We propose a novel tie-oriented model for longitudinal event network data. The generating mechanism is assumed to be a multivariate Poisson process that governs the onset and repetition of yearly observed events with two separate intensity functions. We apply the model to a network obtained from the yearly dyadic number of international deliveries of combat aircraft trades between 1950 and 2017. Based on the trade gravity approach, we identify economic and political factors impeding or promoting the number of transfers. Extensive dynamics as well as country heterogeneities require the specification of semiparametric time-varying effects as well as random effects. Our findings reveal strong heterogeneous as well as timevarying effects of endogenous and exogenous covariates on the onset and repetition of aircraft trade events.
\end{abstract}

Keywords: arms trade network; combat aircraft; longitudinal network analysis; relational event model

\section{Introduction}

Network data capture information on relations between actors. The various types of links between actors in the network encompass stable ties associated with some duration. For example, in political science, military alliance agreements are active for a certain number of years (Cranmer et al., 2012; Leeds, 2019). A different type of link consists of instantaneous bilateral events-like hostile actions measured in real-time (Boschee et al., 2018). Note that instantaneous events can be viewed as the limit case of stable ties if the duration of these ties goes to zero (Butts \& Marcum, 2017). While instantaneous events can happen anytime, they are not always observable in a high resolution of time. Under these circumstances, we can count the instantaneous events occurring in a given time interval, which implies a network-based counting process. We define the respective class of processes as a multivariate counting process that simultaneously guides all dyadic interactions within an event network and dedicate this article to its analysis. Comprehensive monographs and survey articles on statistical network analysis are available in Kolaczyk (2009), Kolaczyk (2017), Goldenberg et al. (2010), Lusher et al. (2012). Recent overviews of dynamic network modeling can be found in Fritz et al. (2020), Kim et al. (2018).

In real-life applications, most networks exhibit dynamics, that is, structural changes over time are driven by endogenous and exogenous determinants, being covariates that capture the present or past network dependencies and additional information external to the evolution of the network,

(C) The Author(s), 2021. Published by Cambridge University Press. This is an Open Access article, distributed under the terms of the Creative Commons Attribution-NonCommercial-NoDerivatives licence (http://creativecommons.org/licenses/by-nc-nd/4.0/), which permits non-commercial re-use, distribution, and reproduction in any medium, provided the original work is unaltered and is properly cited. The written permission of Cambridge University Press must be obtained for commercial re-use or in order to create a derivative work. 
respectively. One way to conceive the generating process of networks is to represent it as a discrete Markov chain, where the realized path consists of the observed networks, and the state space is the set of all observable networks. The transition probabilities defining the chain are given by a distribution over all possible networks (Robins \& Pattison, 2001). For stable ties, this view results in the temporal exponential random graph model (TERGM, Hanneke et al., 2010).

Alternatively, we can perceive the networks as evolving over time, guided by a continuous Markov process (Holland \& Leinhardt, 1977). In this case, network dynamics are often modeled by the stochastic actor-oriented model (SAOM, Snijders, 1996) or in the case of instantaneous events with a precise time stamp by the relational event model (REM) as proposed by Butts (2008). Although modern sensory technology eases the collection of such fine-grained data (Lazer et al., 2009), exact continuous information is usually not obtainable for every observed event. In our case, for example, data on the transactions of combat aircraft trades are collected yearly, but the exact time point of each event (e.g., day of delivery) is impossible to verify (SIPRI, 2019). Therefore, instead of observing instantaneous events, we only protocol the counts of events during given intervals. Consequently, the resulting event data can be comprehended as valued networks, weighted by the count of events that happened within the given intervals. Though the body of literature on dynamic network models is steadily growing, the consideration of valued dynamic networks is less developed and mainly limited to cross-sectional analyses (see Desmarais \& Cranmer, 2012; Krivitsky, 2012; Robins et al., 1999; Krivitsky et al., 2009).

In this article, we introduce a tie-oriented model for the analysis of network-based event data. Tie-oriented models assume a bilateral intensity governing the occurrence of events within a dyad, as opposed to actor-oriented models suggested by Stadtfeld (2012) and extended in Hoffman et al. (2020), Stadtfeld et al. (2017). This approach partitions the intensity into an egocentric senderspecific intensity and a probability selecting the receiver conditional on the sender along the lines of the discrete choice model of McFadden (1973). To represent the dynamic evolution of the network-based process, we start with a framework that operates in continuous time at the tie level. Because the ranking of events in our application is not unique due to the lack of exact time stamps, standard REMs (Butts, 2008; Vu et al., 2011) cannot be readily applied. Therefore, we develop our model under the assumption that the exact ordering of aircraft deliveries within the window of a year is unknown and uninformative. Given that a perennial interplay between policymakers of the involved countries as well as a lengthy order process preludes each trade, this assumption seems reasonable (Snijders, 2017).

Our approach extends existing models in multiple ways. Firstly, we generalize the separable decomposition of network dynamics differentiating between the formation and dissolution of ties introduced by Krivitsky \& Handcock (2014), Holland \& Leinhardt (1977). In particular, we extend the separable decomposition to event and count data instead of the continuous specification given in Krivitsky \& Handcock (2014) and Holland \& Leinhardt (1977), where solely binary and durable ties are regarded. Thereby, we enhance recently introduced windowed effects by Stadtfeld et al. (2017). Furthermore, we propose a semiparametric specification and use penalized B-splines to obtain flexible time-varying coefficients (Eilers \& Marx, 1996). In a similar approach, Bauer et al. (2021) employ non-linear effects to investigate the collaboration between inventors through joint EU patents. Kreiß et al. (2019) propose a nonparametric model with time-varying coefficients that necessitates time-continuous observations, although focusing on the estimator's properties as the number of actors goes to infinity. To capture latent actor-specific heterogeneity, we include random effects for each actor in the network differentiating between the sender and receiver of events. As an application case, we focus on the strategically most crucial international deliveries of weapons, namely combat aircraft from 1950 to 2017 (Forsberg, 1994; SIPRI, 2020a). Combat aircraft comprises all "unmanned aircraft with a minimum loaded weight of $20 \mathrm{KG"} \mathrm{(SIPRI,} \mathrm{2020b).}$ They are very costly, and the number of units transferred constitutes highly valuable information for military strategists (Forsberg, 1997). Therefore, we propose to focus on yearly unit sales as a substantial quantity. 
The remainder of this article is structured as follows: the next section formally introduces the tie-oriented model based on a network-based counting process together with extensions to separable, time-varying, and random effects and an estimation procedure. Consecutively, we introduce the application case and apply our novel method. The paper concludes with Section 4.

\section{Network-based counting process}

\subsection{A framework for discrete and continuous time event data}

We start by proposing the model for time-continuous event data, which are observed at discrete time points. We use the temporal indicator $\tilde{t} \in \mathcal{T}=[0, T)$ and mathematically define the networkvalued process as a Poisson process on a valued network given by:

$$
N(\tilde{t})=\left(N_{i j}(\tilde{t}) \mid i, j \in\{1, \ldots, n\}\right)
$$

where $n \in \mathbb{N}$ is the total number of actors in the network. Process (1) counts the relational events between all actors in the network during the interval $[0, \tilde{t})$. It is characterized by the networkvalued intensity rate $\tilde{\lambda}(\tilde{t})=\left(\tilde{\lambda}_{i j}(\tilde{t}) \mid i, j \in\{1, \ldots, n\}\right)$. The $(i, j)$ th entry of this intensity is defined as the probability that we observe an instantaneous jump of size 1 in $N_{i j}(\tilde{t})$. Heuristically, this is the probability of the occurrence of a directed event from actor $i$ to $j$ at time point $\tilde{t}$. By definition, we set $\tilde{\lambda}_{i i}(\tilde{t})=0 \forall i \in\{1, \ldots, n\}$ and $\tilde{t} \in \mathcal{T}$.

Assuming the process is observed at discrete time points $t \in\{0, \ldots, T\}$ leads to the timediscrete observations $\boldsymbol{Y}_{t}$, which are defined as cumulated events through:

$$
\boldsymbol{Y}_{t}=\mathbf{N}(t)-\mathbf{N}(t-1) \forall t \in\{1, \ldots, T\}
$$

with $N(0)$ set to 0 . Based on the properties of a Poisson process, these increments follow a matrixvalued Poisson distribution:

$$
\boldsymbol{Y}_{t} \sim \operatorname{Pois}\left(\int_{t-1}^{t} \tilde{\lambda}(\tilde{u}) d \tilde{u}\right) \forall t \in\{1, \ldots, T\}
$$

Given that the exact orderings of events within each observation window are not known and assumed to be uninformative, the integrated intensity on the time interval $(t-1, t]$ simplifies to a constant, so that $\int_{t-1}^{t} \tilde{\lambda}(\tilde{u}) d \tilde{u}=\lambda(t)$ holds. Accordingly, we define the observed values of $\boldsymbol{Y}_{t}$ as $y_{t}$. As a result of Equation (2), the waiting times between subsequent events follow an exponential distribution. Therefore, our model is equivalent to the REM as introduced in Butts (2008) in the special case where $\left\|y_{t}\right\|_{1}=1 \forall t \in\{1, \ldots, T\}$ holds under piece-wise constant intensities.

Generally, we are interested in modeling $\lambda(t)$ conditional on the past network topology and exogenous covariates, which are denoted by $x_{t}$. Covariates can be node-specific (regarding either a feature of the sender or receiver), dyadic (regarding a relation between the sender and receiver), or global (regarding the complete network). Building on a first-order Markov property, we allow the intensity to depend on the past network behavior and exogenous covariates through:

$$
Y_{i j, t} \sim \operatorname{Pois}\left(\lambda_{i j}\left(t, y_{t-1}, x_{t-1}\right)\right) \forall t \in\{1, \ldots, T\} ; i, j \in\{1, \ldots, n\}, i \neq j
$$

This is equivalent to the assumption of dyadic independence of events to occur in each time interval given information on the past and exogenous covariates. Similar assumptions were made by Lebacher et al. (2021) in the context of separable TERGMs (Krivitsky \& Handcock, 2014). Almquist \& Butts (2014) justify this method for network panel data where little simultaneous dependence between possible ties is present. For our application to the international combat aircraft trades, this can be legitimized by the long time span of aircraft trades between the order and delivery of units. ${ }^{1}$ 
Accordingly, we specify the intensity in time-varying semiparametric form through:

$$
\lambda_{i j}\left(t, \boldsymbol{y}_{t-1}, \boldsymbol{x}_{t-1}\right)=\lambda_{0}(t) \exp \left\{\theta(t)^{\top} s_{i j}\left(\boldsymbol{y}_{t-1}, \boldsymbol{x}_{t-1}\right)\right\} \forall t \in\{1, \ldots, T\}
$$

where $\lambda_{0}(t)$ is the baseline intensity, $s_{i j}\left(\boldsymbol{y}_{t-1}, \boldsymbol{x}_{t-1}\right)$ is a multidimensional vector consisting of network statistics and theoretically derived exogenous covariates in $t-1$. We discuss different specifications of the statistics in Section 3 where we describe the application case in more detail. The coefficient vector $\theta(t)$ is possibly time-varying and needs to be estimated from the data.

In many application cases, compositional changes of the actor set occur. To compensate for this phenomenon in the model, we include indicator functions similar to risk indicators in timeto-event analysis (Kalbfleisch \& Prentice, 2002). To be specific, we multiply the intensity by an indicator function, determining whether actors $i$ and $j$ are both present in the network at time $t$ :

$$
\lambda_{i j}\left(t, y_{t-1}, x_{t-1}\right)=\mathbb{I}\left(i, j \in \mathcal{R}_{t}\right) \lambda_{0}(t) \exp \left\{\theta(t)^{\top} s_{i j}\left(y_{t-1}, x_{t-1}\right)\right\} \forall t \in\{1, \ldots, T\}
$$

with $\mathcal{R}_{t}$ denoting the set of actors partaking in the network at time point $t$. By including the indicator functions $\mathbb{I}\left(i, j \in \mathcal{R}_{t}\right)$, we decompose our observed network into a stochastic and deterministic component. The latter component consists of structural zeros at time point $t$ in the modeled network between all actors where at least one side is not present. With these actor set changes, the possible range of the network statistics changes as well, leading to values which are not scaled coherently for a comparison across years. To solve this issue, we divide all network statistics by their maximal value to allow for a cohesive interpretation.

\subsection{Extensions}

\subsubsection{Separability assumption}

Interaction patterns are commonly substantially different for already linked and still unlinked actors. To adequately capture this characteristic, Holland \& Leinhardt (1977) proposed a processbased model for binary ties taking the values " 0 " or " 1 " by two separate intensity functions. One intensity toggles entries from "0" to " 1 " (formation of ties) and another one from " 1 " to "0" (dissolution of ties). Thereby, separate and potentially differential effects of statistics depending on previous interaction behavior are enabled. This model, henceforth called separable model, was later adopted to the SAOM by incorporating a so-called gratification function (Snijders \& van Duijn, 1997; Snijders, 2003) and to the TERGM by extending it to the separable TERGM (Krivitsky \& Handcock, 2014). However, one should keep these separable models apart from the separability condition introduced in Almquist \& Butts (2014). In the following, we combine the framework of REMs with the separability approach as coined by Krivitsky \& Handcock (2014).

More specifically, we postulate two different conditions for the network-based process under which the effect of all covariates changes. One condition governs events between unlinked actors and is characterized by the onset intensity. The second condition only regards events among actors that already interacted with each other and is driven by the repetition intensity. In accordance with the Markov assumption specified in Equation (4), we define the onset intensity at time $t$ to control all events which did not occur in $y_{t-1}$. Accordingly, the repetition intensity drives the events that did occur at least once in $y_{t-1}$. This can be incorporated by splitting the intensity into two conditional intensities:

$$
\lambda_{i j}\left(t, \boldsymbol{y}_{t-1}, \boldsymbol{x}_{t-1}\right)= \begin{cases}\lambda_{i j}^{+}\left(t, \boldsymbol{y}_{t-1}, \boldsymbol{x}_{t-1}\right), & \text { if } y_{i j, t-1}=0 \\ \lambda_{i j}^{-}\left(t, \boldsymbol{y}_{t-1}, \boldsymbol{x}_{t-1}\right), & \text { if } y_{i j, t-1}>0\end{cases}
$$

where $\lambda_{i j}^{+}\left(t, y_{t-1}, x_{t-1}\right)$ and $\lambda_{i j}^{-}\left(t, y_{t-1}, x_{t-1}\right)$ are defined along the lines of Equation (4) and specified by the corresponding time-varying parametric effects $\theta^{+}(t)$ and $\theta^{-}(t)$ jointly represented by $\theta(t)=\left(\theta^{+}(t), \theta^{-}(t)\right)$. The possibly overlapping vectors of statistics are denoted accordingly as $s_{i j}^{+}\left(\boldsymbol{y}_{t-1}, \boldsymbol{x}_{t-1}\right)$ and $s_{i j}^{-}\left(\boldsymbol{y}_{t-1}, \boldsymbol{x}_{t-1}\right)$, respectively. Setting $s_{i j, 0}^{+}\left(\boldsymbol{y}_{t-1}, \boldsymbol{x}_{t-1}\right)=1$ enables the inclusion 
of a time-varying intercept $\lambda_{0}^{+}(t)=\exp \left\{\theta_{0}^{+}(t)\right\}$ in the onset model, this holds similarly for the repetition model. Consecutively, the complete separable model is given by replacing Equation (4) with

$$
\begin{aligned}
& \lambda_{i j}\left(t, \boldsymbol{Y}_{t-1}, \boldsymbol{x}_{t-1}\right)= \exp \left\{\mathbb{I}\left(y_{i j, t}=0\right)\left[\theta^{+}(t)^{\top} s_{i j}^{+}\left(y_{t-1}, x_{t-1}\right)\right]\right. \\
&\left.+\mathbb{I}\left(y_{i j, t}>0\right)\left[\theta^{-}(t)^{\top} s_{i j}^{-}\left(y_{t-1}, x_{t-1}\right)\right]\right\} \\
&=\exp \left\{\theta(t)^{\top} s_{i j}\left(y_{t-1}, x_{t-1}\right)\right\}
\end{aligned}
$$

where $\theta(t)=\left(\theta^{+}(t), \theta^{-}(t)\right)$ and

$$
s_{i j}\left(y_{t-1}, x_{t-1}\right)=\left(\mathbb{I}\left(y_{i j, t}=0\right) \cdot s_{i j}^{+}\left(y_{t-1}, x_{t-1}\right), \mathbb{I}\left(y_{i j, t}>0\right) \cdot s_{i j}^{-}\left(y_{t-1}, x_{t-1}\right)\right)
$$

\subsubsection{Spline-based time-varying effects}

Let the $k$ th component of statistic $s_{i j}\left(\boldsymbol{y}_{t-1}, \boldsymbol{x}_{t-1}\right)$ be defined as $s_{i j, k}\left(\boldsymbol{y}_{t-1}, \boldsymbol{x}_{t-1}\right)$ with the matching coefficient $\theta_{k}(t)$. We expand each component $\theta_{k}(t)$ in a semiparametric way by replacing it with a B-spline basis function (see de Boor, 2001). More specifically, we place equidistant knots on a grid in $\mathcal{T}$, where the number of knots can be chosen relatively high (Kauermann \& Opsomer, 2011). In principle, we could choose individual grids for each component of $\theta(t)$, but for the sake of a simple notation, we select the same one for all covariates. We now rewrite each coefficient as:

$$
\theta_{k}(t)=B(t) \alpha_{k} \forall k \in\{0, \ldots, K\}
$$

where $B(t) \in \mathbb{R}^{q}$ is the B-spline basis evaluated at $t$ and $\alpha_{k} \in \mathbb{R}^{q}$ denotes the corresponding coefficient vector. In our context, $q$ constitutes the dimension of the B-spline basis and hence gives the number of separate B-spline bases used for each covariate. To obtain a smooth fit, we penalize the difference of adjacent basis coefficients $\alpha_{k}$ as proposed by Eilers \& Marx (1996). This leads to the overall penalized log-likelihood function:

$$
\ell_{p}\left(\alpha_{0}, \ldots, \alpha_{K}, \gamma_{0}, \ldots, \gamma_{K}\right) \propto \sum_{t=1}^{T} \sum_{i, j \in \mathcal{R}_{t}}\left(y_{i j, t} \log \left(\lambda_{i j, t}\right)-\lambda_{i j, t}\right)-\frac{1}{2} \sum_{k=0}^{K} \gamma_{k} \alpha_{k}^{\top} D_{k} \alpha_{k}
$$

with $\lambda_{i j, t}=\lambda_{i j}\left(t, \boldsymbol{y}_{t-1}, \boldsymbol{x}_{t-1}\right)$. The penalty results from the quadratic form with penalty matrix $D_{k}$ constructed from pairwise differences of the spline coefficients and $\gamma_{k}$ as the penalty (and hence tuning) parameter. This vector $\gamma=\left(\gamma_{1}, \ldots, \gamma_{K}\right)$ controls the smoothness of the fit and is chosen data based following a mixed model approach as described in detail in Ruppert et al. (2003), see also Wood (2017). The incorporation of a penalization in Equation (9) results in a biased estimator and a so-called bias-variance tradeoff, which is thoroughly discussed for penalized spline smoothing in Ruppert et al. (2003). Kauermann \& Opsomer (2011) extend the theoretical results toward a data-driven finite-sample version, and Kauermann et al. (2009) show that the estimates from Equation (9) are consistent.

\subsubsection{Accounting for nodal heterogeneity}

The specification of the model introduced so far implicitly implies that the nodal heterogeneity is fully captured by the structural statistics $s_{i j}\left(\boldsymbol{y}_{t-1}, \boldsymbol{x}_{t-1}\right)$. As already thoroughly discussed by Thiemichen et al. (2016) or Box-Steffensmeier et al. (2018), this can be considered a questionable assumption. It seems, therefore, advisable to include sender- and receiver-specific random effects to account for unobserved heterogeneity. Let therefore $u_{i}^{S}$ denote a latent sender-specific effect of actor $i$ and $u_{j}^{R}$ the receiver-specific effect of actor $j$. This leads to the heterogeneous intensity

$$
\lambda_{i j}\left(t, \boldsymbol{y}_{t-1}, \boldsymbol{x}_{t-1}, u^{S}, u^{R}\right)=\lambda_{i j}\left(t, \boldsymbol{y}_{t-1}, \boldsymbol{x}_{t-1}\right) \exp \left\{u_{i}^{S}+u_{j}^{R}\right\} \forall t \in\{1, \ldots, T\}
$$


We assume $u^{S}=\left(u_{1}^{S}, \ldots, u_{n}^{S}\right)^{\top} \sim N\left(0, I_{n} \tau_{S}^{2}\right)$ and $u^{R}=\left(u_{1}^{R}, \ldots, u_{n}^{R}\right)^{\top} \sim N\left(0, I_{n} \tau_{R}^{2}\right)$ with $I_{n}$ as the $n \times n$ identity matrix. The expression $\lambda_{i j}\left(t, \boldsymbol{y}_{t-1}, \boldsymbol{x}_{t-1}\right)$ may be specified through Equation (4) or (7). Conditional on the random effects $u^{S}$ and $u^{R}$, the distributional assumption (3) still holds

$$
\begin{aligned}
& Y_{i j}(t) \mid u^{S}, u^{R} \sim \operatorname{Pois}\left(\lambda_{i j}\left(t, \boldsymbol{y}_{t-1}, \boldsymbol{x}_{t-1}, u^{S}, u^{R}\right)\right) \\
& \forall t \in\{1, \ldots, T\} ; i, j \in\{1, \ldots, n\}, i \neq j
\end{aligned}
$$

where $\lambda_{i j}\left(t, y_{t-1}, \boldsymbol{x}_{t-1}, u^{S}, u^{R}\right)$ is specified in Equation (10).

\subsection{Estimation}

The vector-valued function $\theta(t)=\left(\theta^{+}(t), \theta^{-}(t)\right)$ is estimated by finding the argument maximizing the penalized likelihood resulting from Equation (11) and viewing the penalty on coefficient vector $\alpha$ as an improper prior distribution. This leads to a generalized additive mixed model, which is extensively discussed in Wood (2017), Ruppert et al. (2003), Ruppert et al. (2009). To leverage the advanced optimization techniques proposed for this model class, we initially calculate all covariates $s_{i j}\left(\boldsymbol{y}_{t-1}, \boldsymbol{x}_{t-1}\right)$ for each actor-tuple and at each point in time. By doing that, we transform the data into a generalized version of the so-called counting-process representation, which is known from time-to-event analysis (Tutz \& Schmid, 2016; Friedman, 1982; Whitehead, 1980). For each snapshot of the event network at time point $t$, this procedure generates a design matrix of $\left|\mathcal{R}_{t}\right|$ conditionally independent observations with a target variable $y_{i j, t}$ expressing the number of events that occurred between a specific tuple of actors and covariates given by $s_{i j}\left(\boldsymbol{y}_{t-1}, \boldsymbol{x}_{t-1}\right)$.

For the estimation, we use the versatile $\mathrm{R}$ package mgcv (Wood, 2017, version 1.8-31). Thereby, we follow Wood et al. (2017) who enhance the pseudo-quasi-likelihood method by Breslow \& Clayton (1993) for the analysis of larger data sets. The main extensions are threefold:

(1) The tuning parameters $\gamma$ are not estimated until convergence in each iteration of the estimation procedure but updated by only one Newton step.

(2) Efficient methods for computing the matrix cross-products in each iteration are run in parallel (Li \& Wood, 2020).

(3) The covariates are discretized along a marginal grid. Hence, the design matrices for the smooth covariates take significantly less memory.

Wood et al. (2017) describe the method in detail as it is implemented in the function bam of the already mentioned $\mathrm{R}$ package. Well-calibrated frequentist confidence bands for the estimated function $\theta(t)$ are guaranteed by Bayesian large sample properties (Wood, 2006).

\section{Application}

\subsection{Data}

So far, quantitative work on the international arms trade utilizing statistical network analysis has mostly been restricted to binarized networks. Here, the occurrence of a trade relationship between two countries in a specific year was modeled conditional on endogenous and exogenous statistics by the gravity model of trade by employing TERGMs and extensions of it (Lebacher et al., 2021; Thurner et al., 2019). Contrary, Lebacher et al. (2020) fit a network disturbance model on the yearly aggregated trend indicator values (SIPRI, 2020b) of the international arms trades, maintaining the valued character of deliveries. All these contributions rely on data provided by the Stockholm International Peace Research Institute (SIPRI, 2020a), and they consider each type of major conventional weapons indiscriminately.

In the following, we concentrate on the counts of combat aircraft deliveries, as reported in the SIPRI data, where each combat aircraft delivery is perceived as an event. We focus on the 


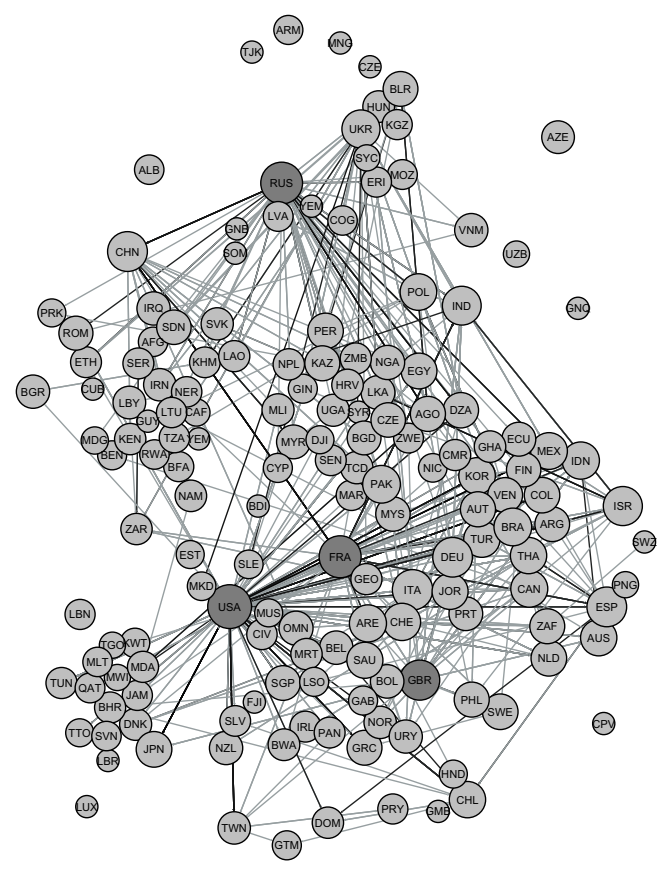

Years $1995-2000$

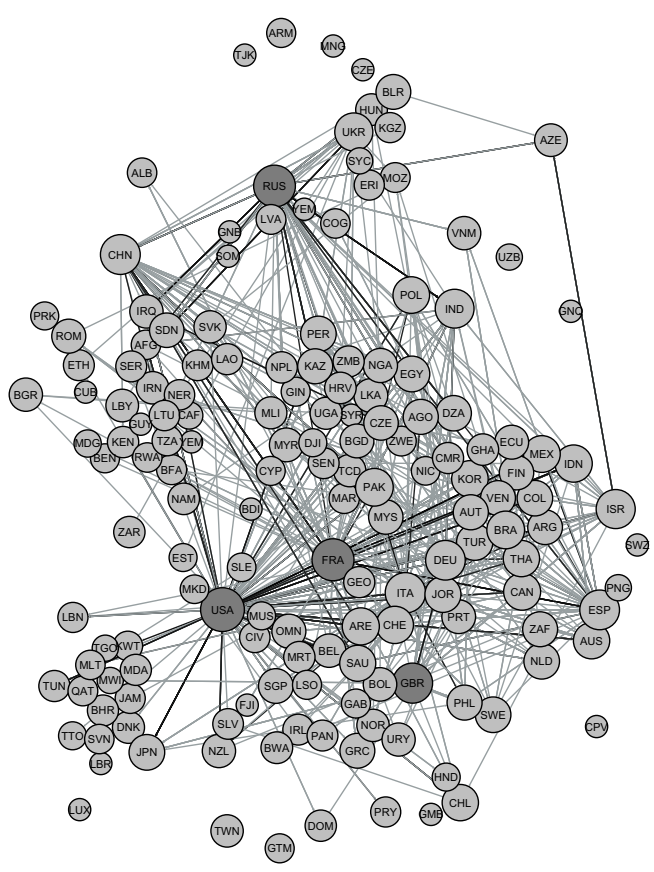

Years $2012-2017$

Figure 1. The international network of combat aircraft trades in two periods. Node size is proportional to the sum of involved deals and the grey-scale of each tie indicates the aggregated amount of deals in the specific time frame. The labels of the nodes are the ISO3 codes of the respective countries. The four major sender countries are drawn in a darker shade.

transfers of aircraft because these weapon systems usually incorporate the highest technological sophistication. Therefore, they are being restricted to close allies. Furthermore, they are of crucial strategic importance for international deterrence and counterinsurgency in intrastate conflict (Hoeffler \& Mérand, 2016; Mehrl \& Thurner, 2020). Lastly, their sizes and cost make the available data highly reliable (Forsberg, 1994, 1997). Previous research on combat aircraft trade was limited to the quantitative analysis of a small subset of countries or fighter programs (Hoeffler \& Mérand, 2016; Vucetic, 2011; Vucetic \& Nossal, 2012). Contrasting these endeavors, we take a global point of view on the combat aircraft trade. Here, a closer look at the data reveals how countries commonly partition major deals with their stable trade partners into multiple deliveries occurring over the span of several years. For instance, the United States and Japan signed a deal in 1984 comprising 32 quantities of aircraft, which were realized between 1988 and 2016. The additional information provided by this segmentation of trade deals into isolated deliveries would be lost when only regarding binarized networks. ${ }^{2}$

Two examples of the network representing aggregated events over 6 years are depicted in Figure 1. Generally, the networks exhibit a structure with hubs around the United States (USA), Russia (RUS), France (FRA), and United Kingdom (UK). Coincidentally, this set of countries also demonstrate the highest average hub-scores over time (Kleinberg, 1999). Analog to the distribution of the in- and out-degrees in binary networks, we can examine the distribution of the concatenated in- and outgoing event counts for all years. We call the respective statistics in- and out-count, although they are equivalent to the generalized degree proposed by Opsahl et al. (2010). The empirical distribution of those statistics enables a better understanding of the topology of the observed networks. Figure 2(a) suggests a strong centralization in the outward event count distribution. Some countries are the sender of up to 130 deliveries in one year. Still, on average, $82 \%$ 
(a)

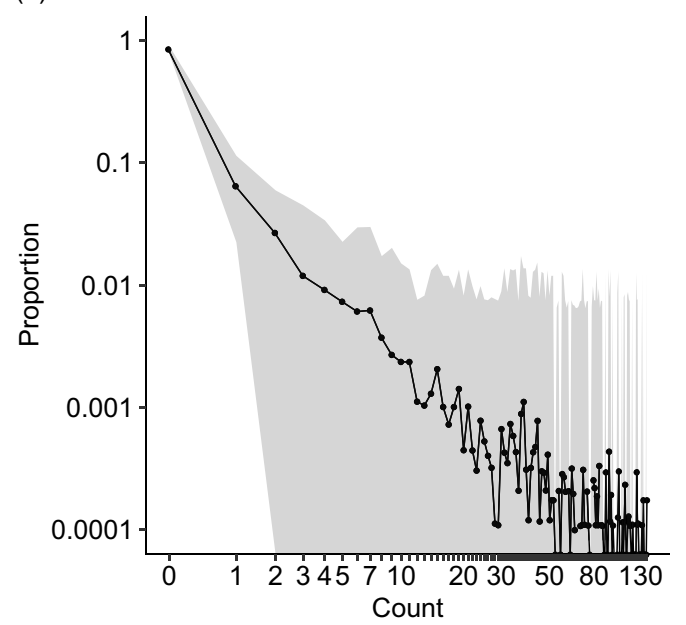

(b)

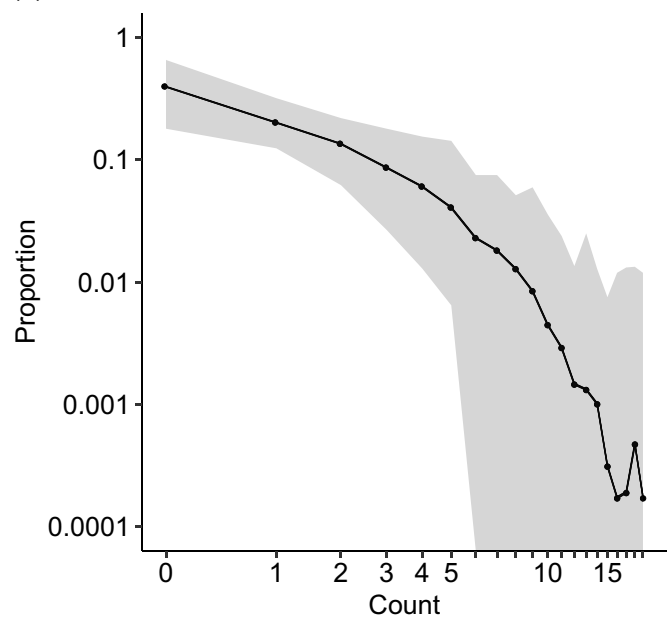

Figure 2. Distributions of the Out- and In-Counts for all included countries concatenated over all years. The shaded area represents the minimum and maximum of the observed values. Both graphs are represented on a logarithmic scale.

of the countries do not export. The inward count distribution is not as skewed and centralized, as shown in Figure 2(b). Nonetheless, the mode of the distribution is still at zero.

\subsection{Model specification}

We now employ the outlined model to the international combat aircraft trade network spanning from 1950 to 2017. The event networks are observed yearly. In this context, $y_{i j, t}$ denotes the number of observed combat aircraft units delivered in year $t$ between country $i$ and $j$ and its distribution follows from Equation (3). Given this information, we estimate the time-continuous intensities of all country-dyads, which are per assumption governed by the repetition intensity if the respective countries traded in the previous year and by the onset intensity otherwise as defined in Equation (7). ${ }^{3}$ All network actors are countries, and an event represents the delivery of combat aircraft between them. To appropriately capture interdependencies of the observed event counts, we incorporate a wide range of endogenous statistics, whose mathematical representation is given in Table 1 and visualized in Figure 3. Generally, we define all non-binary structural statistics to be bounded between 0 and 100 to guarantee a consistent interpretation independent of the varying network size and prevent the implied autoregressive counting process from unrealistic behavior (Gjessing et al., 2010).

As already investigated in multiple applications (Snijders, 2003; Newman et al., 2002), the degree structure plays a crucial role in the observed event network. In the case of directed events, the in- and out-degree of a country determine its relative location in the network (Wasserman \& Faust, 1994). In our application, the degrees reflect the number of different countries with whom a specific country had at least one transaction in a particular year as an importer (in-degree) and exporter (out-degree). To reveal the impact of these measures on the intensity of observing an event, we include four degree-related statistics concerning the sender and receiver in our specification, as illustrated in Figure 3(a)-(d). For instance, one can interpret a positive effect of the sender's out-degree as the tendency to trade with countries that are already sending a lot in the previous year.

Besides degree-based statistics, Holland \& Leinhardt (1971), Davis (1970) highlight the role of triangular structures in networks. When adapted to event relations, it refers to the change in intensity of an event between countries $i$ and $j$, if they are indirectly connected by an additional 
Table 1. Mathematical formulations of the structural covariates as calculated for $s_{i j}\left(\boldsymbol{y}_{t-1}, \boldsymbol{x}_{t-1}\right)$. The number of countries that are present in the network at time point $t$ is denoted by $n_{t}$. The identifying letters concern the respective graphical illustrations in Figure 3

\begin{tabular}{ll}
\hline Name & Mathematical representation \\
\hline (a) In-degree sender & $\frac{100}{n_{t}-1} \sum_{h=1}^{n} \mathbb{I}\left(y_{h i, t-1}>0\right)$ \\
\hline (b) In-degree receiver & $\frac{100}{n_{t}-1} \sum_{h=1}^{n} \mathbb{I}\left(y_{h j, t-1}>0\right)$ \\
\hline (c) Out-degree sender & $\frac{100}{n_{t}-1} \sum_{h=1}^{n} \mathbb{I}\left(y_{i h, t-1}>0\right)$ \\
\hline (d) Out-degree receiver & $\frac{100}{n_{t}-1} \sum_{h=1}^{n} \mathbb{I}\left(y_{j h, t-1}>0\right)$ \\
\hline (e) Transitivity & $\frac{100}{n_{t}-2} \sum_{h=1}^{n} \mathbb{I}\left(y_{i h, t-1}>0\right) \mathbb{I}\left(y_{h j, t-1}>0\right)$ \\
\hline (f) Shared supplier & $\frac{100}{n_{t}-2} \sum_{h=1}^{n} \mathbb{I}\left(y_{h i, t-1}>0\right) \mathbb{I}\left(y_{h j, t-1}>0\right)$ \\
\hline (g) Reciprocity & $\mathbb{I}\left(y_{j i, t-1}>0\right)$ \\
\hline
\end{tabular}

(a) In-Degree Sender

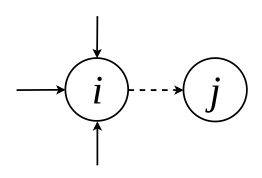

(b) In-Degree Receiver

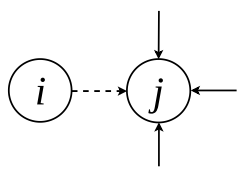

(c) Out-Degree Sender

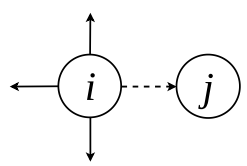

(d) Out-Degree Receiver

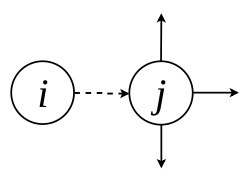

(e) Transitivity

(f) Shared Supplier

(g) Reciprocity
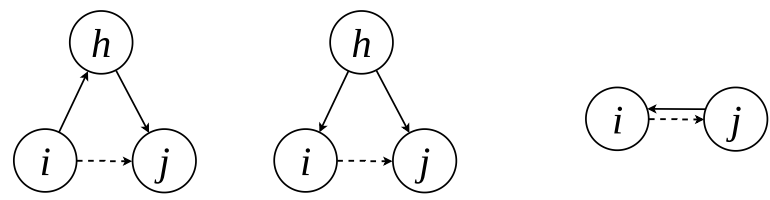

Figure 3. Graphs consisting of three arbitrary actors $i, j$, and $h$ that illustrate the included triangular and dyadic covariates in the first row. Dashed arrows represent the event that is modeled and solid arrows in $t-1$.

two-path, i.e., third country. Since the aircraft deliveries between countries are directed, there are multiple ways to define two-paths. We incorporate two triadic structures: transitivity, Figure 3(e), and shared supplier, Figure 3(f). While transitivity in an event network suggests that already having observed a delivery from country $i$ to $k$ and $k$ to $j$ affects the intensity of an event from $i$ to $j$, the shared supplier mechanism reflects the tendency toward trading with countries that import combat aircraft from a common exporter. These triangular structures were the only variants found to be relevant for the trade of combat aircraft. Likewise, we control for reciprocity, which is the tendency of countries to respond to previous events directed at them, Figure $3(\mathrm{~g})$.

Political economy models of arms trade (Levine et al., 1994; Thurner et al., 2019) as well as the gravity model of arms trade guide the selection of appropriate exogenous covariates. Thurner et al. (2019), Akerman \& Seim (2014) included the dyadic distance in kilometers between the capitals of country $i$ and $j$ as well as the logarithmic gross domestic product (GDP in US \$) of the sender and receiver countries as covariates in the model. Pamp et al. (2018), Lebacher et al. (2021) emphasize the impact of military expenditures as a proxy for the Newtonian power of attraction, which we include in logarithmic form as a sender- and receiver-specific covariate. The respective yearly data were collected by SIPRI (2019) in US \$ and combined by Nordhaus et al. (2012) with data from Singer et al. (1972). We use this combined data set but employ linear interpolation if at least $60 \%$ of the time series for a specific country is observed. Moreover, we incorporate two dyadic variables controlling whether country $i$ and $j$ signed an alliance treaty or are similar to each other in terms of their regimes in power, following Martínez-Zarzoso \& Johannsen (2019), Thurner et al. (2019). The alliance treaty obligations and provisions project identified military alliance 
(a)

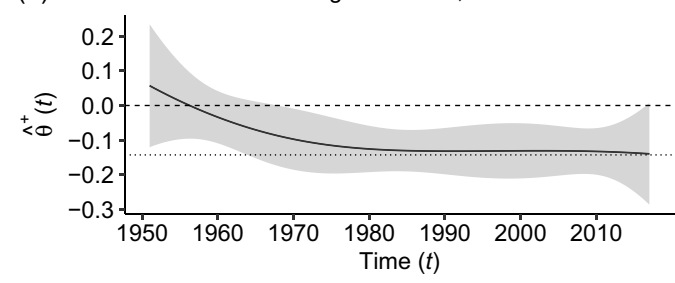

(c)

In-Degree Receiver, Onset

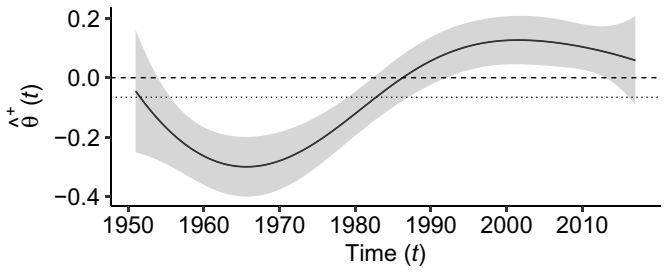

(e)

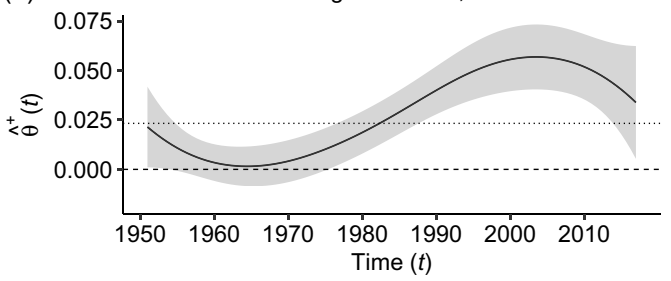

(g)

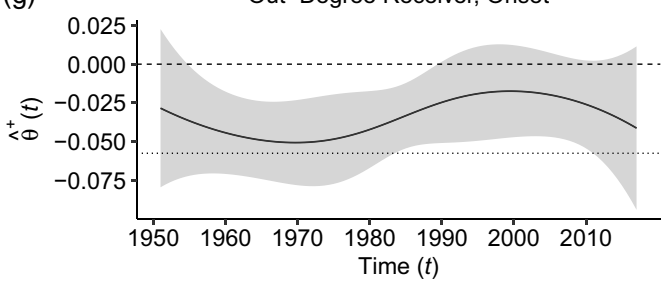

(b)

In-Degree Sender, Repetition

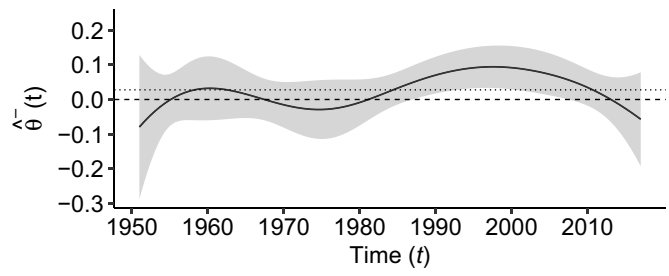

(d)

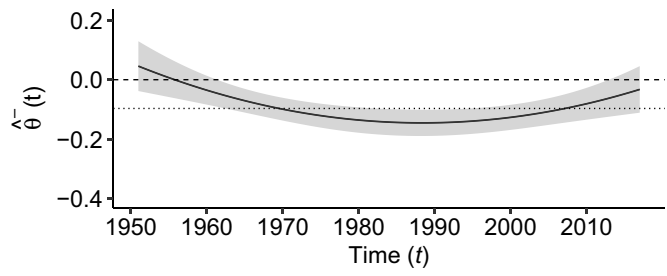

(f)

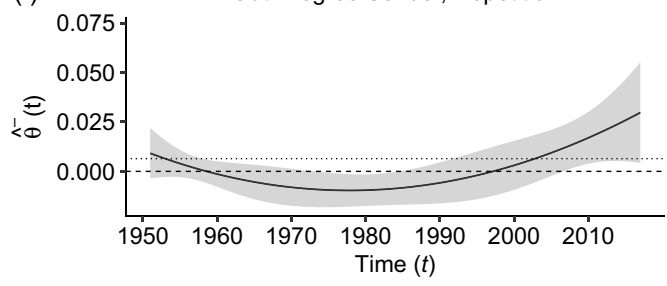

(h)

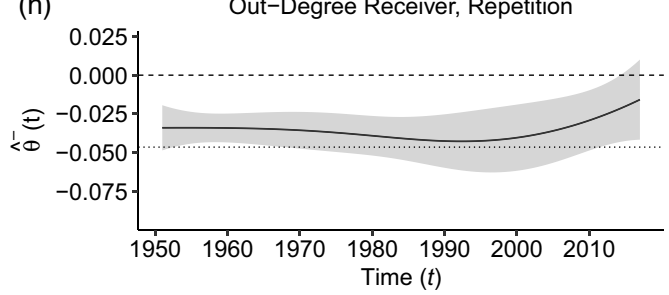

Figure 4. Results of endogenous statistics relating to centrality. The shaded area indicates the $95 \%$ confidence bands of the estimates and the dotted horizontal lines represent the time-constant parameters.

agreements (Leeds, 2019) and we operationalize regime dissimilarity by the absolute difference in the Polity IV scores of countries $i$ and $j$ (Marshall, 2017). This measure indicates all countries' year-wise regime characteristics and takes values from -10 (strongly autocratic) to 10 (strongly democratic). Thus, the absolute differences lie between 0 (strong similarity) and 20 (strong dissimilarity) for each country-dyad and year. The sources and used period of all incorporated exogenous covariates are described in more detail in the Supplementary Material.

\subsection{Results}

\subsubsection{Fixed effects}

In Figures $4-7$, the full results of the time-varying estimates are given accompanied by alternative time-constant coefficients as dotted horizontal lines. The latter are obtained by setting $\theta(t) \equiv$ $\theta$. All exponentially transformed estimates at a specific point in time can be interpreted (ceteris paribus) as the multiplicative change of the intensity (6) corresponding to the effect of covariates in relative risk models (Kalbfleisch \& Prentice, 2002). Therefore, an effect estimated at zero does not change the relative risk of an event to happen, but positive or negative coefficients lead to a 

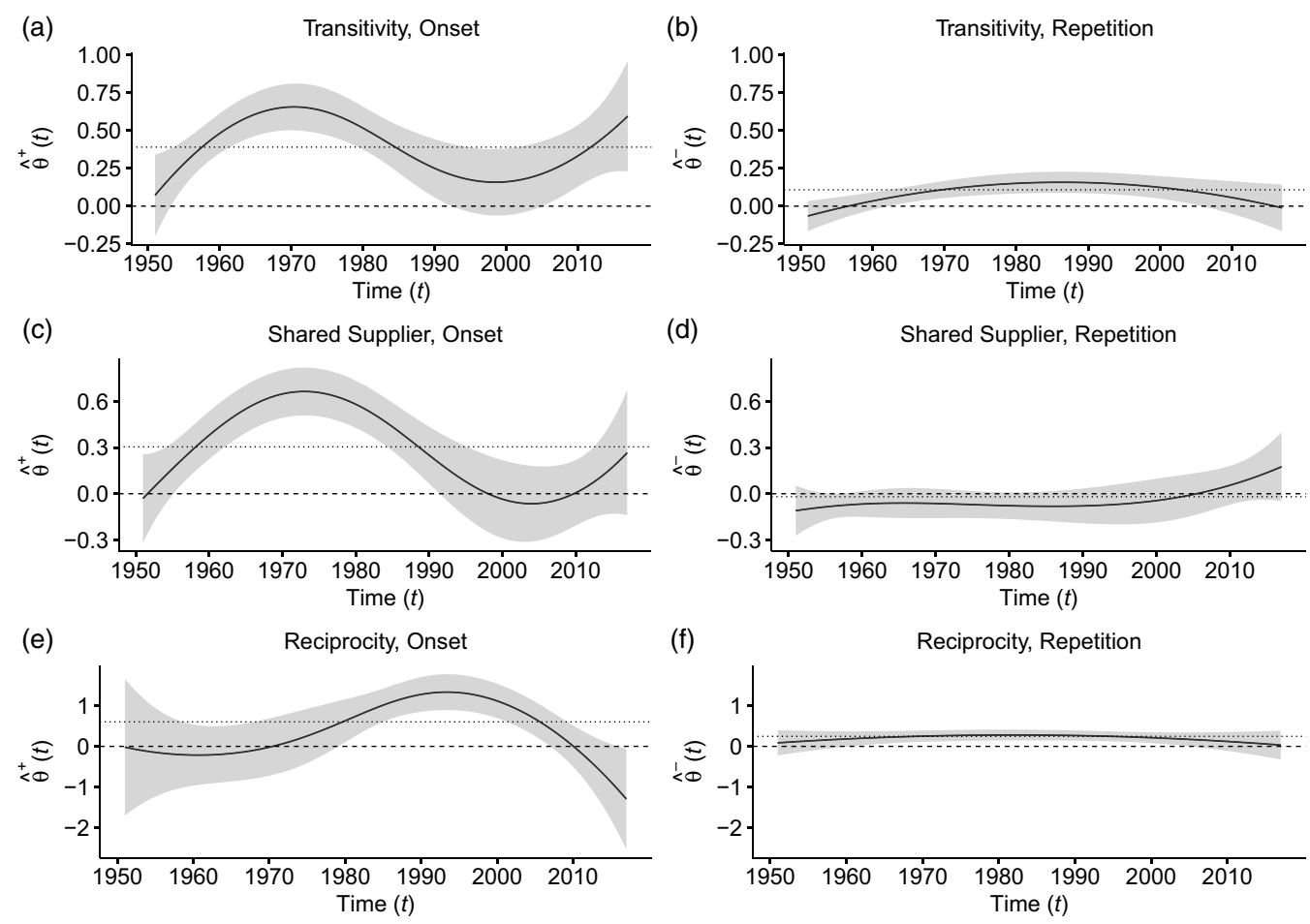

Figure 5. Results of endogenous statistics relating to past dyadic interaction and clustering. The shaded area indicates the $95 \%$ confidence bands of the estimates and the dotted horizontal lines represent the time-constant parameters.

higher or lower relative risk of the event to occur, respectively. Additionally, an event's occurrence is equivalent to the increment of one in the counts of aircraft units since one event represents a combat aircraft delivery in our application case.

From simple inspection, it can be concluded that in all cases, time-varying coefficients are carrying completely different information as compared to time-constant coefficients. This is evidence of the necessity to account for the multiple systemic changes within the international aircraft market during the considered time interval. From a statistical point of view, the time-varying effects can also be underpinned by a lower cAIC value when compared to time-constant effects (see Section 3.4 for additional details on the cAIC).

Moreover, we observe different shapes of the curves of the time-varying coefficients when comparing onset and repetition conditions leading to the conclusion that the import of all covariates on these two separate conditions is different.

Time-varying effects relating to the degree structure are shown in Figure 4. Figure 4(a) indicates a steady negative influence of the sender's in-degree in the onset condition from around 1965 onward. It can be concluded that the count of dyadic events is lower if the sender's in-degree is high, which may be justified by the observation that only a small subset of countries is adequately equipped to produce and export aircraft. This technological possibility, in turn, increases selfsufficient behavior, thus alleviating the need for additional imports. Contrary, in the repetition condition, the in-degree of the receiver exhibits a positive effect for the post-Cold War period from 1990 to 2010, Figure 4(b). Otherwise, the effect is insignificant. Concerning the receiver, a negative effect of the in-degree can be observed from 1950 to 1980 in the onset model, Figure $4(\mathrm{c})$. When proceeding to deliver aircraft, the receiver's in-degree effect is similar to the sender's in-degree effect, Figure 4(d). For the sender's out-degree, the effect in the onset model is negative until around 1980 and thereupon positive. In the latter case, the effect mirrors a higher tendency 
(a)

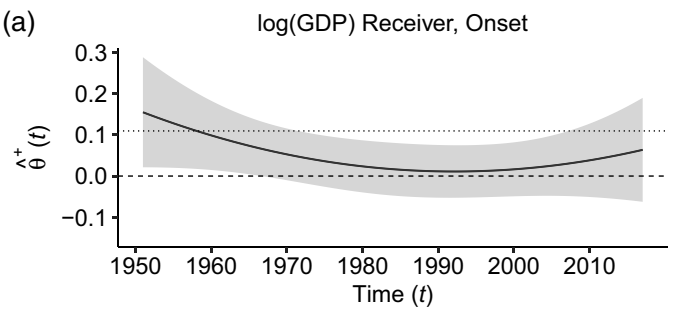

(c)

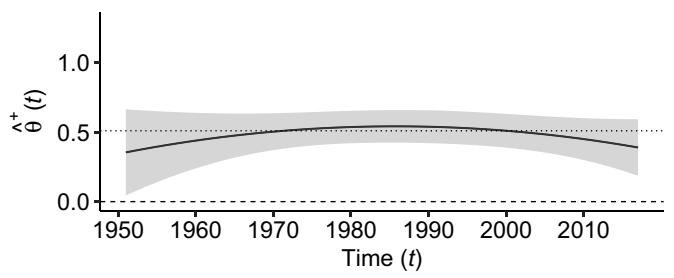

(e)

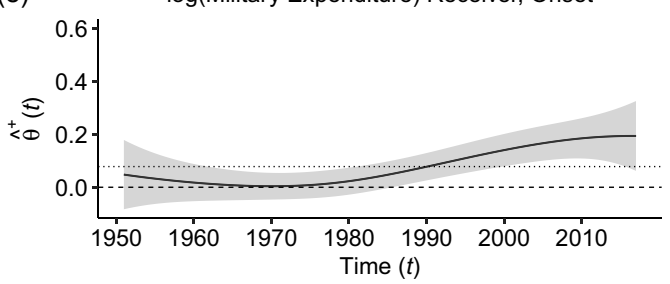

(g)

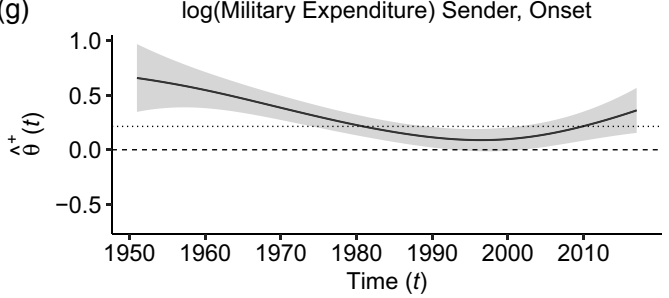

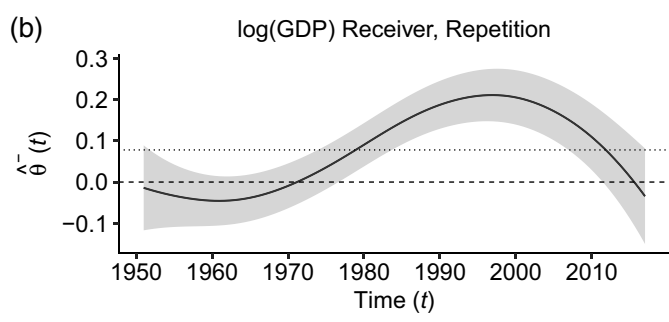

(d)

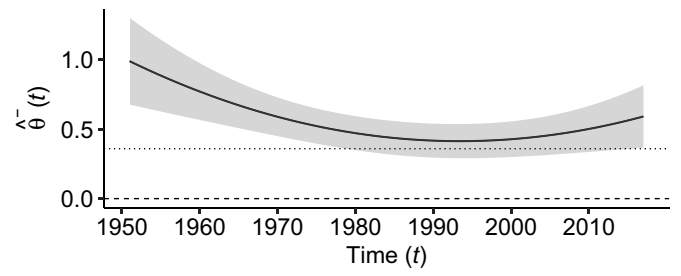

(f) $\quad \log ($ Military Expenditure) Receiver, Repetition

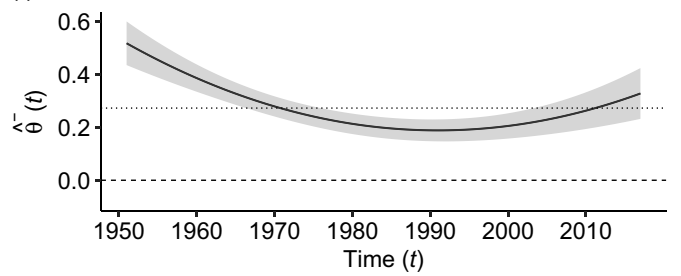

(h)

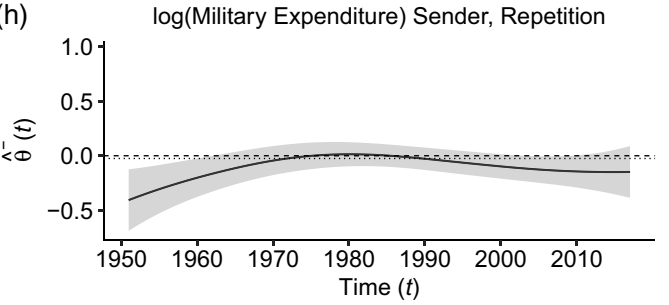

Figure 6. Results of exogenous statistics relating to economic factors. The shaded area indicates the $95 \%$ confidence bands of the estimates and the dotted horizontal lines represent the time-constant parameters.

of delivering combat aircraft if the sender is already a prolific exporter country. During the entire observational period, we observe that receivers are not senders themselves, thus exhibiting low out-degrees, Figure 4(g) and (h). This behavior does not depend on the condition of the dyadic intensity.

The specified triadic structures play a substantial role during the Cold War. Afterwards, the impact disappears but is again strengthened after 2000 under the onset condition, Figure 5(a) and (c). In particular, an increasing number of indirect transitive connections between country $i$ and $j$ results in a greater count of aircraft deliveries between 1950 and 1990. Similarly, receiving combat aircraft from the same third country increases the unit sales between the receivers during the Cold War period, Figure 5(c). A possible consequence of this process is the strengthening of a block structure. For a consecutive delivery, the triadic effects are less pronounced, and in the case of shared suppliers, Figure 5(d), constantly insignificant. The count of reciprocal events, on the other hand, raises trade from 1990 to 2005, Figure 5(e). This result may be a consequence of an international market opening after the Soviet Union's fall, leading to multiple emergent countries. If the relationship is maintained, reciprocal events are encouraged throughout the period of observation, although to a smaller degree, Figure 5(f). 
(a)

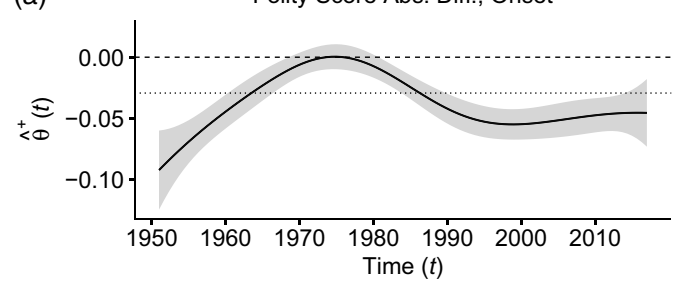

(c)

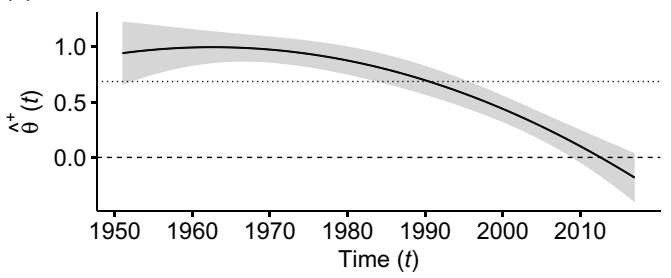

(e)

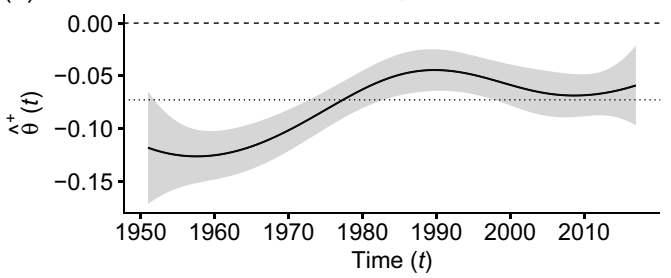

(b)

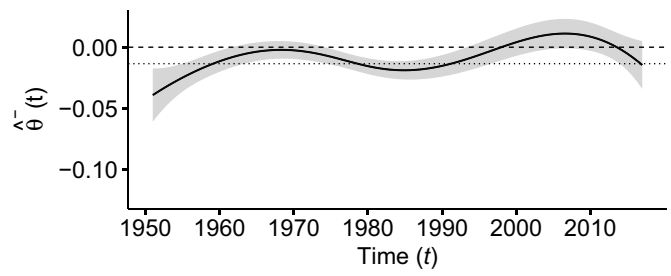

(d)

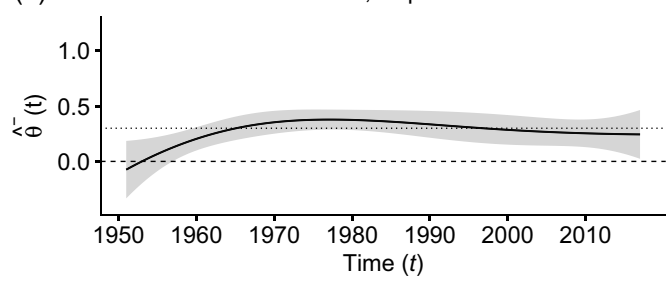

(f)

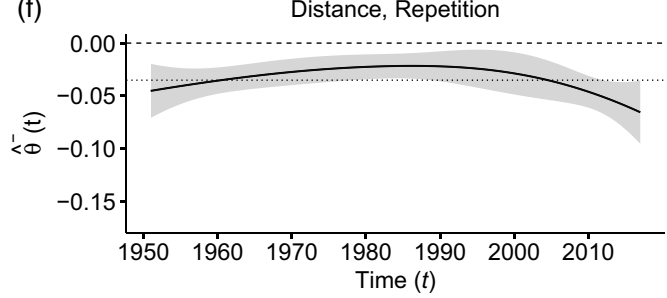

Figure 7. Results of exogenous statistics relating to political, security, and geographical factors. The shaded area indicates the $95 \%$ confidence bands of the estimates and the dotted horizontal lines represent the time-constant parameters.

While the logarithmic GDP of the receiver has a relatively weak positive influence when starting a trade relation, Figure 6(a), its repetition is only affected after the end of the Cold War, Figure 6(b). On the sender-side, the estimates of both models are constantly positive, Figure 6(c) and (d). In contrast to the effect in the onset model, the sender's logarithmic GDP has a higher effect from 1950 to 1980 in the repetition condition. Moreover, the military expenditure of the receiver is one of the main drivers in this model, Figure 6(f). Here, higher military spending of possible sender countries augments the count of receiving combat aircraft deliveries, specifically during the 1950s. Conversely, the exogenous covariate only slowly gains attention in the onset condition after the Cold War, Figure 6(e). While the effect of the military expenses of the sender stays overall positive when delivering aircraft for the first time, it inhibits it to be repeated in the next year, Figure 6(g) and (h).

The findings in Figure $7(\mathrm{a})$ and (b) indicate that similar regimes are overall more likely to start trading combat aircraft. Only at the height of the Cold War from 1970 to 1980, the effect is estimated at approximately 0, Figure 7(a). The strength of the effect is less salient in the repetition condition than in the onset condition of our model, Figure $7(\mathrm{~b})$. Furthermore, the timevarying coefficients discover a steadily decreasing influence of beginning to transact with allies, Figure 7(c). This finding suggests evidence of the overall deteriorating importance of international alliances in combat aircraft transactions if they did not trade in the previous year. We do not observe a similar downward trend when repeating an event, Figure 7(d). Lastly, a larger distance between the respective capitals generally hinders events from occurring, Figure 7(e) and (f). Therefore, countries tend to trade with spatially more close than distant partners. This may be caused by the relatively lower transportation cost and is in line with the expectations of the gravity model of trade (Martínez-Zarzoso \& Johannsen, 2019; Thurner et al., 2019, see corrigendum). 


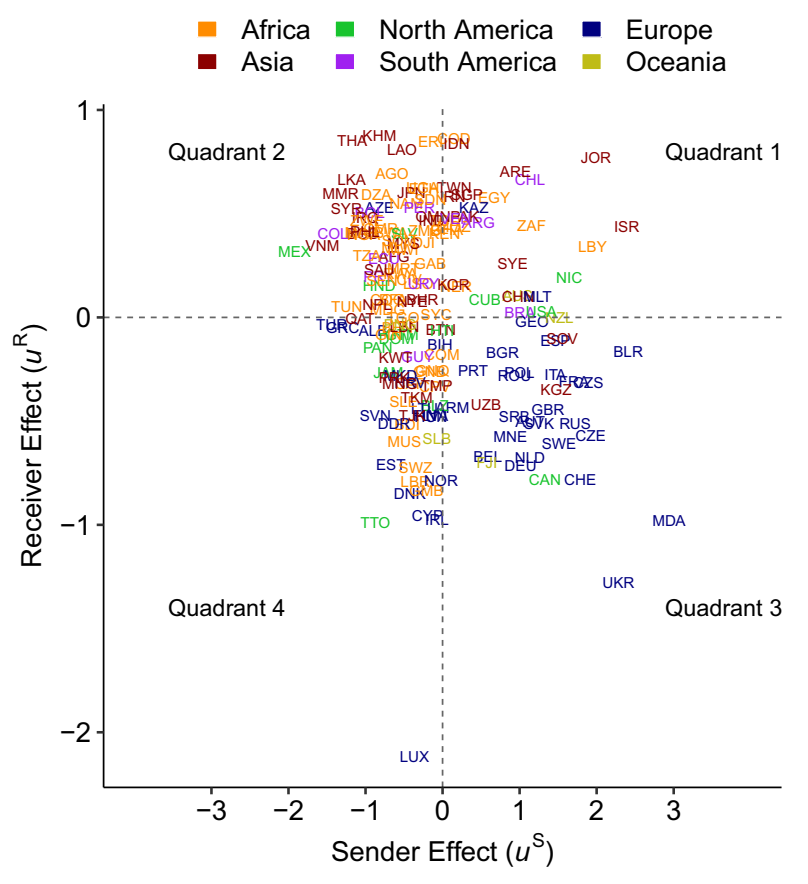

Figure 8. Country-specific random sender and receiver effects. The drawn label represents the respective ISO3 code of the represented country.

\subsubsection{Random effects}

The random effects permit an extended analysis of the unexplained heterogeneity in the model. More precisely, the random effects express country-specific deviations from an overall behavioral trend captured by the time-varying effects. Additionally, they correct the countries' repeated measurements as simultaneous senders and receivers of events in each year. The model introduced in Section 2 comprises two country-specific random effects for all countries as a sender and receiver of combat aircraft deliveries. The results are given in Figure 8 and visualized on a world map in Figure 9.

In the first quadrant of Figure 8, countries with a positive random sender and receiver effect are shown. This composition of random effects suggests that the respective countries are senders and receivers of more combat aircraft events than marginally expected. Countries in the Middle East, for example, Israel (ISR), Libya (LBY), and Jordanian (JOR), are allocated to this group.

Negative sender but positive receiver effects are identified for countries in South-East Asia (Thailand (THA), Cambodia (KHM), Laos (LAO), Myanmar (MYR), and Sri Lanka (LKA)). Compared to the average behavior, these countries are somewhat reluctant as senders and confident as receivers of combat aircraft deliveries. The latent sender effect of Mexico (MEX) is the most negative coefficient estimated. This suggests Mexico's reliance on the import of combat aircraft, although its high economic status would imply additional participation in the event network as a sender.

The third quadrant contains all countries, which were less active than expected as a sender and receiver of events. This strand of countries is either economically strong, yet exhibiting a passive trading behavior, for example, Luxembourg (LUX), or relatively poor and missing preconditions to send or receive weapons, for example, Trinidad and Tobago (TTO).

Lastly, a negative random coefficient regarding receiving arms is mostly associated with European countries. The corresponding sender effect is positive. Hence, these countries are 
(a)

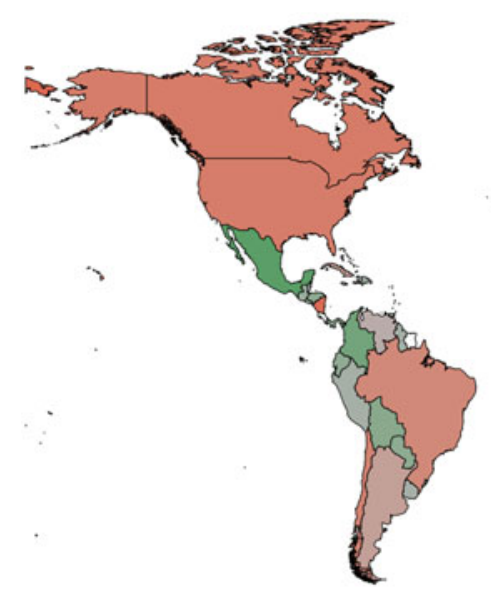

Random Sender Effects

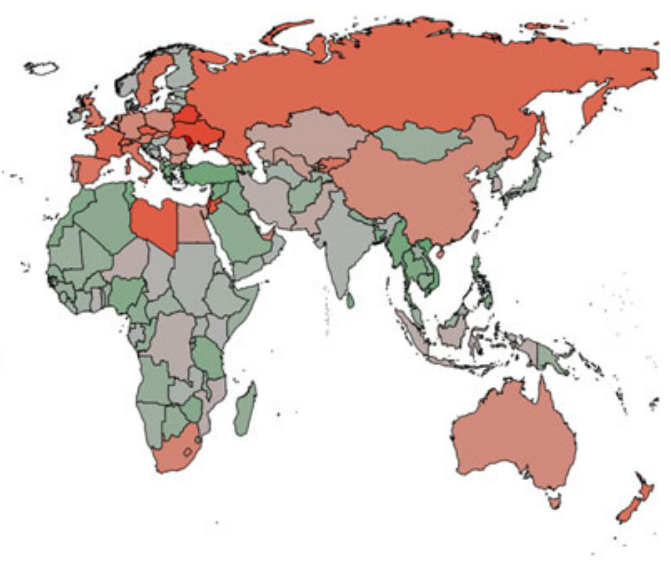

(b)

Random Receiver Effects
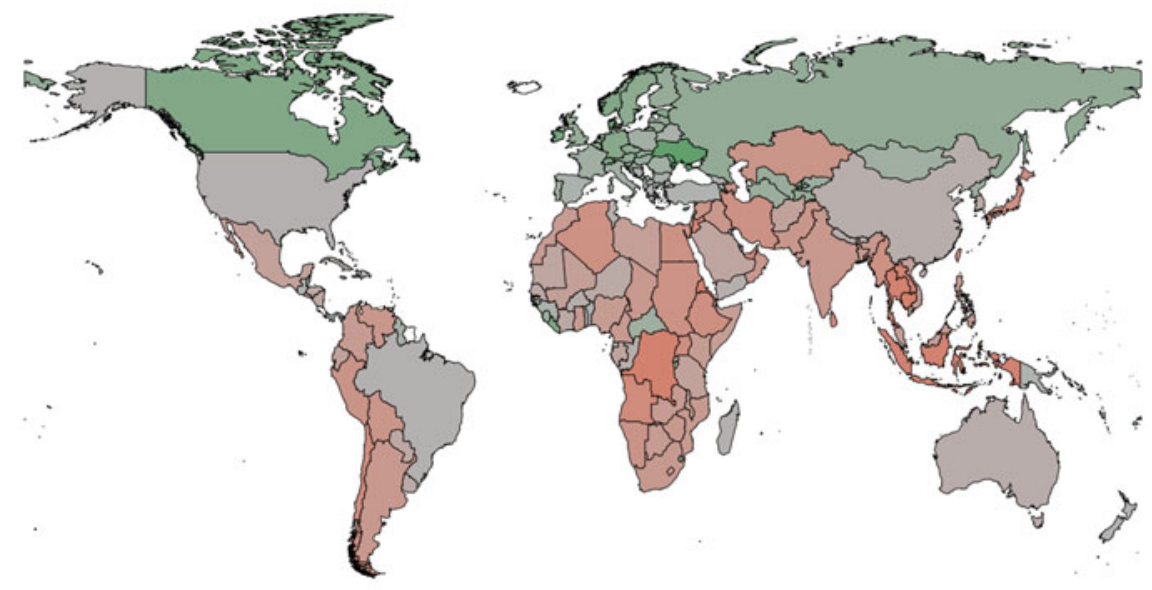

$-3-2-1001223$

Figure 9. Random country-specific sender (a) and receiver (b) effects. The layout represents the borders as of 2020.

situated in the fourth quadrant of Figure 8. The East European countries Moldova (MDA), Ukraine (UKR), and Belarus (BLR) have the highest positive sender effect paired with relatively low receiver effects.

In terms of continent-wide tendencies, we locate Africa in the first three quadrants. South America is principally assigned to the first and second quadrant. Asia, Oceania, and North America are more dispersed and exhibit less homogeneous country behavior.

\subsection{Model comparison and assessment}

We compare the estimated model to alternative specifications, which are chosen to reflect all subsequent extensions of Section 2.2 and are indicated in Table 2. Model 1 includes all effects linearly without the separable extension. This is we assume that $\theta(t) \equiv \theta$ and omit the separation of the statistics $s_{i j}\left(y_{t-1}, x_{t-1}\right)$ into $s_{i j}^{+}\left(y_{t-1}, x_{t-1}\right)$ and $s_{i j}^{-}\left(y_{t-1}, x_{t-1}\right)$. This separability is added in 
Table 2. Specifications of the compared models and resulting corrected AIC (CAIC) values

\begin{tabular}{ccccc} 
& Separability & Time-varying effects & Random effects & cAIC \\
\hline Model 1 & & & $84,622.47$ \\
Model 2 & $\checkmark$ & $\checkmark$ & $65,614.86$ \\
Model 3 & $\checkmark$ & $\checkmark$ & & $63,174.49$ \\
Model 4 & $\checkmark$ & & $\checkmark$ & $59,717.77$ \\
\hline
\end{tabular}

Model 2 according to Section 2.2.1. Model 3 includes time-varying coefficients as introduced in Section 2.2.2. Lastly, Model 4 is the model whose findings were presented in Section 3.3. Hence, also random effects are taken into account, which are explained in Section 2.2.3.

One way to compare these models is by means of information criteria, that is, the Akaike information criterion (AIC, Akaike, 1974). As already discussed in the context of linear mixed models (Greven \& Kneib, 2010) and generalized mixed models (Saefken et al., 2014), the usage of the conditional or marginal AIC does not appropriately incorporate the uncertainty of estimating the covariance parameters of the random effects (in our application $\tau_{S}^{2}$ and $\tau_{R}^{2}$ ). Therefore, we utilize a corrected conditional AIC proposed by Wood et al. (2016). The resulting cAIC values are given in Table 2 and indicate a superior model fit when all extensions introduced in Section 2.2 are included.

We assess the selected Model 4 with a graphical tool proposed by Hunter et al. (2008) for general network models. The procedure's basic idea is to evaluate whether networks randomly generated according to the estimated network model at hand conserve pre-specified characteristics of the observed network reasonably well. In our particular case, we simulate yearly increments of our network counting process from Equation (11) and consider the result as a count-valued network. However, most network statistics commonly used for this assessment are solely defined for binary networks. Therefore, we propose a suite of novel statistics for our application case. To detect whether our model adequately replicates possible over- or underdispersion in the count data, we rely on the statistics from rootograms, that is, the empirical and simulated frequencies of the counts in the networks. For general regression tasks involving count data, rootograms were proposed by Kleiber \& Zeileis (2016) and date back to Tukey (1977). Usually, one compares the square-root-transformed observed and expected frequencies of the target variable. However, in our application, we substitute the square-root transformation with a log transformation due to the high percentage of zeros and use the simulated rather than expected frequencies to fit into the framework of Hunter et al. (2008). Secondly, we investigate to what extent the performance of our model is stable over the time frame we analyze. To do so, we compute the clustering coefficient for weighted networks as proposed by Opsahl \& Panzarasa $(2009)^{4}$ for the yearly networks $\mathbf{y}_{t}$. Besides, we examine the average in-count per year, which is directly related to the average count per year. In the Supplementary Material, we show how the distribution of the observed counts of in- and outgoing events given in Figure 2 is reproduced in the simulated networks and provide the mathematical formulations of all statistics.

Figure 10 shows the variability of all specified statistics computed for all 1,000 simulated networks through boxplots and displays the average value by a blue triangle. Red lines indicate the observed measurements. We can infer from Figure 10(a) that the estimated model captures even high event counts between countries averaged over the entire period. At the same time, our proposed model is capable of representing the yearly clustering as well as the average in-count, see Figure 10(b) and (c). Therefore, we gather that the performance of the proposed model is consistently good throughout the observational period. 
(a) Rootogram

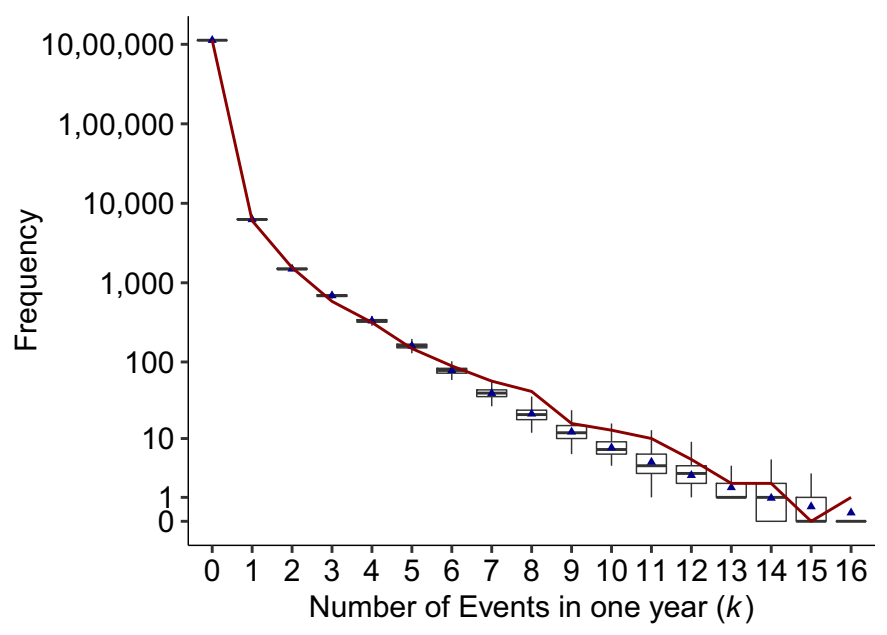

(b)

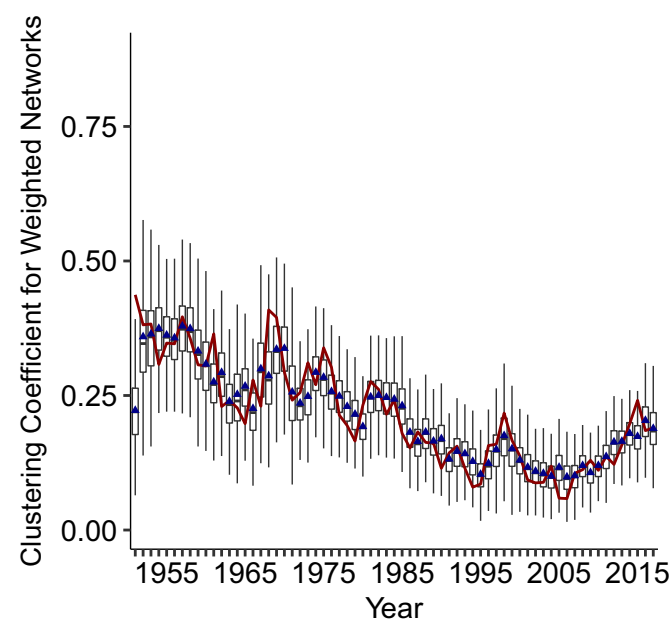

(c)

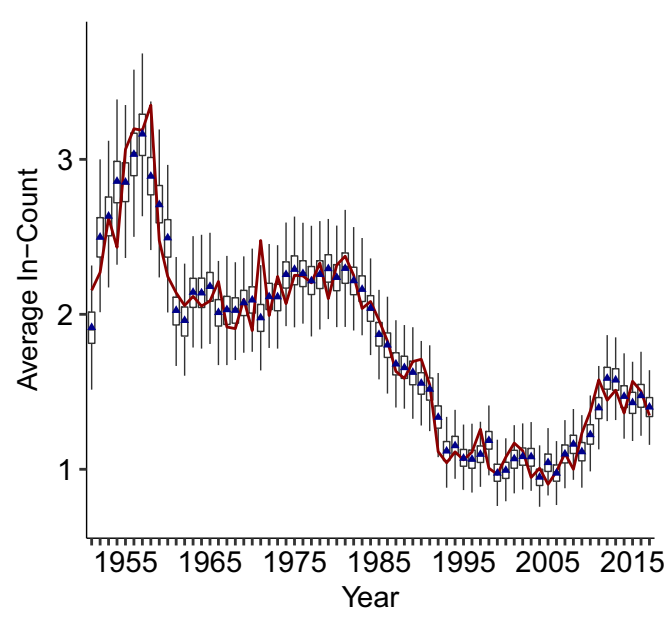

Figure 10. Comparison of the observed and simulated frequencies of the dyadic event counts (a), weighted clustering coefficients over time (b), and average in-count over time (c). The red lines indicate the observed values of each statistic, whereas the boxplots are the result of drawing 1,000 networks according to Equation (11) and the blue triangles the average values.

\section{Conclusion}

We introduced a novel model for the analysis of relational event data. Originating in a counting process operating in continuous time that we only observe at specific time points, we derived a tie-level intensity, whose parameters can be estimated according to the maximum likelihood principle. Extensions to separable models, which govern the onset and repetition of events by two functions, and the incorporation of time-varying and random coefficients are given. Eventually, we applied the procedure to the international combat aircraft network from 1950 to 2017. By doing that, we use the additional information provided by the counts of yearly aircraft deliveries to estimate a time-continuous intensity, contrary to existing work on binarized networks. Moreover, the separability detects fundamentally different processes governing the onset and repetition of event relationships, while the time-varying effects uncover a systemic change during the Cold War period. Furthermore, we identified triangular network statistics and the sender's economic 
nodal covariates as the principal drivers of the onset condition of the proposed intensity. Here, a decaying effect of bilateral military alliances became apparent. For the repetition condition, this effect remained consistently positive, and the receiver's high military expenditure was shown to be the driving force. Finally, the random effects enable a visual comparison of the unexplained heterogeneity between the modeled countries (Figure 9) and correct the estimates for repeated measurements as well as possible overdispersion.

Supplementary materials. For supplementary material for this article, please visit http://dx.doi.org/10.1017/nws.2021.9

Acknowledgments. We thank the anonymous reviewers for their careful reading and constructive comments.

Financial support. The project was supported by the European Cooperation in Science and Technology [COST Action CA15109 (COSTNET)]. We also gratefully acknowledge funding provided by the German Research Foundation (DFG) for the project KA 1188/10-1 and TH 697/9-1: International Trade of Arms: A Network Approach. Furthermore, the work has been partially supported by the German Federal Ministry of Education and Research (BMBF) under Grant No. 01IS18036A. The authors of this work take full responsibilities for its content.

Conflict of interest. None.

\section{Notes}

1 We further provide a descriptive analysis in the Supplementary Material to demonstrate high positive auto-correlation of the endogenous covariates between consecutive years; therefore, they are a reliable proxy of simultaneous dependence.

2 In the Supplementary Material, we deliver the results regarding alternative models for the data. Overall, there is no relevant difference to the findings presented subsequently.

3 As a robustness check, we compare different time frames to define which events are driven by the onset and repetition intensity, for example, having delivered combat aircraft the last one or two years in the Supplementary Material.

$4 \mathrm{We}$ opt for the variant of the statistic that aggregates triplets of event counts within a year via the arithmetic mean.

\section{References}

Akaike, H. (1974). A new look at the statistical model identification. IEEE Transactions on Automatic Control, 19(6), 716-723. Akerman, A., \& Seim, A. L. (2014). The global arms trade network 1950âĂș2007. Journal of Comparative Economics, 42(3), 535-551.

Almquist, Z. W., \& Butts, C. T. (2014). Logistic network regression for scalable analysis of networks with joint edge/vertex dynamics. Sociological Methodology, 44(1), 273-321.

Bastian, M., Heymann, S., \& Jacomy, M. (2009). Gephi: An open source software for exploring and manipulating networks. In Third international AAAI conference on weblogs and social media.

Bauer, V., Harhoff, D., \& Kauermann, G. (2021). A smooth dynamic network model for patent collaboration data. In AStA advances in statistical analysis.

Boschee, E., Lautenschlager, J., O’Brien, S., Shellman, S., \& Starz, J. (2018). ICEWS automated daily event data. Retrieved from https://doi.org/10.7910/DVN/QI2T9A (visited 2019-09-23).

Box-Steffensmeier, J. M., Christenson, D. P., \& Morgan, J. W. (2018). Modeling unobserved heterogeneity in social networks with the frailty exponential random graph model. Political Analysis, 26(1), 3-19.

Breslow, N. E., \& Clayton, D. G. (1993). Approximate inference in generalized linear mixed models. Journal of the American Statistical Association, 88(421), 9-25.

Butts, C. T. (2008). A relational event framework for social action. Sociological Methodology, 38(1), 155-200.

Butts, C. T., \& Marcum, C. S. (2017). A relational event approach to modeling behavioral dynamics. In A. Pilny, \& M. S. Poole (Eds.), Group processes (pp. 51-92). Cham: Springer.

Cranmer, S. J., Desmarais, B. A., \& Menninga, E. J. (2012). Complex dependencies in the alliance network. Conflict Management and Peace Science, 29(3), 279-313.

Csardi, G., \& Nepusz, T. (2006). The igraph software package for complex network research. InterJournal, Complex Systems, 1695(5), 1-9.

Davis, J. A. (1970). Clustering and hierarchy in interpersonal relations: Testing two graph theoretical models on 742 sociomatrices. American Sociological Review, 35(5), 843-851.

de Boor, C. (2001). A practical guide to splines. New York: Springer.

Desmarais, B. A., \& Cranmer, S. J. (2012). Statistical inference for valued-edge networks: The generalized exponential random graph model. PLoS ONE, 7(1), 1-12. 
Eilers, P. H. C., \& Marx, B. D. (1996). Flexible smoothing with B-splines and penalties. Statistical Science, 11(2), 89-102.

Forsberg, R. (1994). The arms production dilemma: Contraction and restraint in the world combat aircraft industry. Cambridge: MIT Press.

Forsberg, R. (1997). The contraction of the world military aircraft industry. In M. Kaldor, B. Vashee, \& G. Scheder (Eds.), The end of military fordism: Restructuring the global military sector. London: Bloomsbury.

Friedman, M. (1982). Piecewise exponential models for survival data with covariates. Annals of Statistics, 10(1), 101-113.

Fritz, C., Lebacher, M., \& Kauermann, G. (2020). Tempus volat, hora fugit: A survey of tie-oriented dynamic network models in discrete and continuous time. Statistica Neerlandica, 74(3), 275-299.

Gjessing, H. K., Røysland, K., Pena, E. A., \& Aalen, O. O. (2010). Recurrent events and the exploding Cox model. Lifetime Data Analysis, 16(4), 525-546.

Goldenberg, A., Zheng, A. X., Fienberg, S. E., \& Airoldi, E. M. (2010). A survey of statistical network models. Foundations and Trends in Machine Learning, 2(2), 129-233.

Greven, S., \& Kneib, T. (2010). On the behaviour of marginal and conditional AIC in linear mixed models. Biometrika, 97(4), 773-789.

Hanneke, S., Fu, W., \& Xing, E. P. (2010). Discrete temporal models of social networks. Electronic Journal of Statistics, 4, 585-605.

Hoeffler, C., \& Mérand, F. (2016). Buying a fighter jet: European lessons for Canada. Canadian Foreign Policy Journal, 22(3), $262-275$.

Hoffman, M., Block, P., Elmer, T., \& Stadtfeld, C. (2020). A model for the dynamics of face-to-face interactions in social groups. Network Science, 8(S1), 1-22.

Holland, P., \& Leinhardt, S. (1971). Transitivity in structural models of small groups. Comparative Group Studies, 2(2), 107124.

Holland, P., \& Leinhardt, S. (1977). A dynamic model for social networks. The Journal of Mathematical Sociology, 5(1), 5-20.

Hunter, D. R., Goodreau, S. M., \& Handcock, M. S. (2008). Goodness of fit of social network models. Journal of the American Statistical Association, 103(481), 248-258.

Kalbfleisch, J. D., \& Prentice, R. L. (2002). The statistical analysis of failure time data. Hoboken: Wiley.

Kauermann, G., Krivobokova, T., \& Fahrmeir, L. (2009). Some asymptotic results on generalized penalized spline smoothing. Journal of the Royal Statistical Society: Series B (Statistical Methodology), 71(2), 487-503.

Kauermann, G., \& Opsomer, J. D. (2011). Data-driven selection of the spline dimension in penalized spline regression. Biometrika, 98(1), 225-230.

Kim, B., Lee, K. H., Xue, L., \& Niu, X. (2018). A review of dynamic network models with latent variables. Statistics Surveys, $12,105-135$.

Kleiber, C., \& Zeileis, A. (2016). Visualizing count data regressions using rootograms. The American Statistician, 70(3), 296303.

Kleinberg, J. M. (1999). Authoritative sources in a hyperlinked environment. Journal of the ACM, 46(5), 604-632.

Kolaczyk, E. D. (2009). Statistical analysis of network data. Methods and models. New York: Springer.

Kolaczyk, E. D. (2017). Topics at the frontier of statistics and network analysis: (Re)visiting the foundations. Cambridge: Cambridge University Press.

Kreiß, A., Mammen, E., \& Polonik, W. (2019). Nonparametric inference for continuous-time event counting and link-based dynamic network models. Electronic Journal of Statistics, 13(2), 2764-2829.

Krivitsky, P. N. (2012). Exponential-family random graph models for valued networks. Electronic Journal of Statistics, 6 , $1100-1128$.

Krivitsky, P. N, \& Handcock, M. S. (2014). A separable model for dynamic networks. Journal of the Royal Statistical Society: Series B (Statistical Methodology), 76(1), 29-46.

Krivitsky, P. N., Handcock, M. S., Raftery, A. E., \& Hoff, P. D. (2009). Representing degree distributions, clustering, and homophily in social networks with latent cluster random effects models. Social Networks, 31(3), 204-213.

Lazer, D., Pentland, A., Adamic, L., Aral, S., Barabási, A. L., Brewer, D., ... Van Alstyne, M. (2009). Computational social science. Science, 323(5915), 721-723.

Lebacher, M., Thurner, P. W., \& Kauermann, G. (2020). Exploring dependence structures in the international arms trade network: A network autocorrelation approach. Statistical Modelling, 20(2), 195-218.

Lebacher, M., Thurner, P. W., \& Kauermann, G. (2021). A dynamic separable network model with actor heterogeneity: An application to global weapons transfers. Journal of Royal Statistical Society: Series A (Statistics in Society).

Leeds, B. A. (2019). Alliance treaty obligations and provisions (ATOP 4.01). Retrieved from http://www.atopdata.org/ (visited 2019-09-30).

Levine, P., Sen, S., \& Smith, R. (1994). A model of the international arms market. Defence and Peace Economics, 5(1), 1-18.

Li, Z., \& Wood, S. N. (2020). Faster model matrix crossproducts for large generalized linear models with discretized covariates. Statistics and Computing, 30, 19-25.

Lusher, D., Koskinen, J., \& Robins, G. (2012). Exponential random graph models for social networks. Cambridge: Cambridge University Press. 
Marshall, M. G. (2017). Polity IV project: Political regime characteristics and transitions, 1800-2016. Retrieved from http://www.systemicpeace.org/inscrdata.html (visited 2019-09-16).

Martínez-Zarzoso, I., \& Johannsen, F. (2019). The gravity of arms. Defence and Peace Economics, 30(1), 2-26.

McFadden, D. (1973). Conditional logit analysis of qualitative choice behavior. In P. Zarembka (Ed.), Frontiers in econometrics (pp. 105-142). New York: Academic Press.

Mehrl, M., \& Thurner, P. W. (2020). Military technology and human loss in intrastate conflict: The conditional impact of arms imports. Journal of Conflict Resolution, 64(6), 1172-1196.

Newman, M. E. J., Watts, D. J., \& Strogatz, S. H. (2002). Random graph models of social networks. Proceedings of the National Academy of Sciences, 99(S1), 2566-2572.

Nordhaus, W., Oneal, J. R., \& Russett, B. (2012). The effects of the international security environment on national military expenditures: A multicountry study. International Organization, 66(3), 491-513.

Opsahl, T., Agneessens, F., \& Skvoretz, J. (2010). Node centrality in weighted networks: Generalizing degree and shortest paths. Social Networks, 32(3), 245-251.

Opsahl, T., \& Panzarasa, P. (2009). Clustering in weighted networks. Social Networks, 31(2), 155-163.

Pamp, O., Dendorfer, F., \& Thurner, P. W. (2018). Arm your friends and save on defense? The impact of arms exports on military expenditures. Public Choice, 177, 165-187.

Robins, G., \& Pattison, P. (2001). Random graph models for temporal processes in social networks. Journal of Mathematical Sociology, 25(1), 5-41.

Robins, G., Pattison, P., \& Wasserman, S. (1999). Logit models and logistic regressions for social networks: III. Valued relations. Psychometrika, 64(3), 371-394.

Ruppert, D., Wand, M., \& Carroll, R. J. (2003). Semiparametric regression. Cambridge: Cambridge University Press.

Ruppert, D., Wand, M., \& Carroll, R. J. (2009). Semiparametric regression during 2003âĂș2007. Electronic Journal of Statistics, 3(3), 1193-1256.

Saefken, B., Kneib, T., van Waveren, C.-S., \& Greven, S. (2014). A unifying approach to the estimation of the conditional Akaike information in generalized linear mixed models. Electronic Journal of Statistics, 8(1), 201-225.

Singer, J. D., Bremer, S., \& Stuckey, J. (1972). Capability distribution, uncertainty, and major power war, 1820-1965. In B. Russett (Ed.), Peace, war, and numbers (pp. 19-48), vol. 19. Sage.

SIPRI. (2019). Military expenditure database. Retrieved from https://www.sipri.org/databases/milex (visited 2020-09-03).

SIPRI. (2020a). Arms transfers database. Retrieved from https://www.sipri.org/databases/armstransfers (visited 2020-03-09).

SIPRI. (2020b). Arms transfers database: Sources and methods. Retrieved from https://www.sipri.org/databases/ armstransfers/sources-and-methods (visited 2020-03-09).

Snijders, T. A. B. (1996). Stochastic actor-oriented models for network change. Journal of Mathematical Sociology, 21, $149-172$.

Snijders, T. A. B. (2003). Accounting for degree distribution in empirical analysis of network dynamics. In Dynamic social network modeling and analysis: Workshop summary and papers (pp. 146-161). The National Academies Press.

Snijders, T. A. B. (2017). Comment: Modeling of coordination, rate functions, and missing ordering information. Sociological Methodology, 47(1), 41-47.

Snijders, T. A. B., \& van Duijn, M. (1997). Simulation for statistical inference in dynamic network models. In R. Conte, R. Hegselmann, \& P. Terna (Eds.), Simulating social phenomena. Cham: Springer.

Stadtfeld, C. (2012). Events in social networks: A stochastic actor-oriented framework for dynamic event processes in social networks. Ph.D. thesis, KIT.

Stadtfeld, C., Hollway, J., \& Block, P. (2017). Dynamic network actor models: Investigating coordination ties through time. Sociological Methodology, 47(1), 1-40.

Thiemichen, S., Friel, N., Caimo, A., \& Kauermann, G. (2016). Bayesian exponential random graph models with nodal random effects. Social Networks, 46, 11-28.

Thurner, P. W., Schmid, C. S., Cranmer, S. J., \& Kauermann, G. (2019). Network interdependencies and the evolution of the international arms trade. Journal of Conflict Resolution, 63(7), 1736-1764.

Tukey, J. W. (1977). Exploratory data analysis. London: Pearson.

Tutz, G., \& Schmid, M. (2016). Modeling discrete time-to-event data. Cham: Springer.

Vu, D., Hunter, D., Smyth, P., \& Asuncion, A. (2011). Continuous-time regression models for longitudinal networks. In J. Shawe-Taylor, R. Zemel, P. Bartlett, F. Pereira, \& K. Q. Weinberger (Eds.), Advances in neural information processing systems, vol. 24 .

Vucetic, S. (2011). Canada and the F-35: What's at stake? Canadian Foreign Policy Journal, 17(3), 196-203.

Vucetic, S., \& Nossal, K. R. (2012). The international politics of the F-35 Joint Strike Fighter. International Journal, 68(1), $3-12$.

Wasserman, S., \& Faust, K. (1994). Social network analysis: methods and applications. Cambridge: Cambridge University Press.

Whitehead, J. (1980). Fitting cox's regression model to survival data using glim. Journal of the Royal Statistical Society: Series C (Applied Statistics), 29(3), 268. 
Wood, S. N. (2006). On confidence intervals for generalized additive models based on penalized regression splines. Australian and New Zealand Journal of Statistics, 48(4), 445-464.

Wood, S. N. (2017). Generalized additive models: An introduction with R. Boca Raton: CRC press.

Wood, S. N., Li, Z., Shaddick, G., \& Augustin, N. H. (2017). Generalized additive models for gigadata: Modeling the U.K. black smoke network daily data. Journal of the American Statistical Association, 112(519), 1199-1210.

Wood, S. N., Pya, N., \& Säfken, B. (2016). Smoothing parameter and model selection for general smooth models. Journal of the American Statistical Association, 111(516), 1548-1563.

Cite this article: Fritz C., Thurner P.W. and Kauermann G. (2021). Separable and semiparametric network-based counting processes applied to the international combat aircraft trades. Network Science 9, 291-311. https://doi.org/10.1017/nws.2021.9 